Groups Geom. Dyn. 7 (2013), 633-651

DOI $10.4171 / \mathrm{GGD} / 201$
Groups, Geometry, and Dynamics

(C) European Mathematical Society

\title{
Fast growth in the Følner function for Thompson's group $F$
}

\author{
Justin Tatch Moore
}

\begin{abstract}
The purpose of this note is to prove a lower bound on the growth of Følner functions for Richard Thompson's group $F$. Specifically I will prove that, for any finite generating set $\Gamma \subseteq F$, there is a constant $C$ such that $\mathrm{F} \mathrm{l}_{F, \Gamma}\left(C^{n}\right) \geq \exp _{n}(0)$.
\end{abstract}

Mathematics Subject Classification (2010). 20F65, 43A07.

Keywords. Følner function, tower function, Thompson's group, amenable.

\section{Introduction}

In this paper we will study the Følner function for Richard Thompson's group $F$. Recall that a finite subset $A$ of a finitely generated group $G$ is $\varepsilon$-F $\not$ lner (with respect to a finite generating set $\Gamma \subseteq G$ ) if

$$
\sum_{\gamma \in \Gamma}|(A \cdot \gamma) \triangle A|<\varepsilon|A|
$$

where $\triangle$ denotes symmetric difference. The Følner function of $G$ (with respect to $\Gamma)$ is defined by

$$
\mathrm{F} \mathrm{l}_{G, \Gamma}(n)=\min \left\{|A|: A \subseteq G \text { is } \frac{1}{n} \text {-Følner with respect to } \Gamma\right\}
$$

with

$$
\operatorname{Føl}_{G, \Gamma}(n)=\infty
$$

if there is no $1 / n$-Følner set with respect to $\Gamma$. By Følner's criterion, a group $G$ is amenable if and only if its Følner function (with respect to any finite generating set $\Gamma)$ is finite valued.

Thompson's group $F$ has many equivalent definitions; we will use the formulation in terms of tree diagrams defined below. The standard presentation of $F$ is infinite, with generators $x_{i}(i \in \mathbb{N})$ satisfying $x_{i}^{-1} x_{n} x_{i}=x_{n+1}$ for all $i<n$. It is well known, however, that $F$ admits the finite presentation

$$
\left\langle A, B \mid\left[A B^{-1}, A^{-1} B A\right]=\left[A B^{-1}, A^{-2} B A^{2}\right]=\mathrm{id}\right\rangle
$$


(see [2]). Geoghegan conjectured that $F$ is not amenable [5], p. 549, and at present this problem remains open. ${ }^{1}$ The goal of this paper is to establish the following lower bound on the Følner function for $F$.

Theorem 1.1. For every finite symmetric generating set $\Gamma \subseteq F$ there is a constant $C>1$ such that if $A \subseteq F$ is a $C^{-n}$-Følner set with respect to $\Gamma$, then $A$ contains at least $\exp _{n}(0)$ elements. In particular $\mathrm{F}_{F, \Gamma}$ is not eventually dominated $\exp _{p}(n)$ for any finite $p$.

Here $\exp _{p}(n)$ is the $p$-fold composition of the exponential function defined by $\exp _{0}(n)=n$ and $\exp _{p+1}(n)=2^{\exp _{p}(n)}$. If it turns out that $F$ is amenable, then Theorem 1.1 would be a step toward answering (negatively) the following question of Gromov [7], p. 578.

Question 1.2. Is there a primitive recursive function which eventually dominates every Følner function of an amenable finitely presented group?

While it is known that the Følner functions of amenable finitely generated groups can grow arbitrarily fast [4], this is not the case for finitely presented groups (since there are only countably many such groups). See [3] for what can be accomplished via wreath products of $\mathbb{Z}$.

This note is organized as follows. In Section 2, I will review some of the basic definitions associated with $F$ and fix some notational conventions. In Section 3, I will introduce the notion of a marginal set and prove some basic lemmas about them. These are sets which must have small intersections with Følner sets. They play a central role in the proof of the main result of the paper. Section 4 recasts the amenability of $F$ in terms of its partial right action on the finite rooted ordered binary trees $\mathcal{T}$. Section 5 defines an operation on elements of $\mathcal{T}$ which exponentially decreases their size and commutes with the partial right action of $F$. It is shown that the trees which are trivialized by this operation are marginal and it is this that allows the proof of Theorem 1.1.

Acknowledgements. I would like to thank Matt Brin for his careful reading of drafts of this paper, catching errors, and suggesting improvements. In particular, the current formulation of Lemma 3.9 was suggested by him (the original formulation provided a weaker estimate). I would also like to thank the anonymous referee for their very careful reading and helpful comments and suggestions. This research was supported in part by NSF grant DMS-0757507.

\footnotetext{
${ }^{1}$ In fact Thompson himself had studied the question of the amenablity of $F$ already by the early 1970s [9], although the question was not well known until it was independently considered and popularized by Geoghegan.
} 


\section{Notation and background}

I will use [2] as a general reference for Thompson's group $F$, although the reader is warned that the notation in the present paper will differ somewhat from that of [2]. Let $\mathcal{T}$ denote the collection of all finite rooted ordered binary trees. For concreteness, we will view elements $T$ of $\mathcal{T}$ as finite sets of binary sequences which have the following property: for every infinite binary sequence $x$, there is a unique element of $T$ which is an initial part of $x$. Thus an element $T$ of $\mathcal{T}$ is a record of the addresses of the leaves of the tree which it represents. The trivial tree is the set which consists only of the sequence of length 0 .

The collection of finite binary sequences is equipped with the operation of concatenation (denoted by $u^{\wedge} v$ ), the partial order $\subseteq$ of extension (defined by $u \subseteq u^{\wedge} v$ ), and the lexicographic order (denoted by $u<_{\operatorname{lex}} v$ ). Note that $u$ extends $v$ includes the possibility that $u=v$. I will use \langle\rangle to denote the sequence of length $0 . \mathcal{T}$ can also be characterized as being those nonempty finite sets $T$ of binary sequences such that

- no element of $T$ is an initial part of another element of $T$, and

- if $u$ is a binary sequence, then $u^{\wedge} 0$ has an extension in $T$ if and only if $u^{\wedge} 1$ has an extension in $T$.

If $U$ and $V$ are in $\mathcal{T}$, then we will say that $U$ is dominated by $V$ if every element of $U$ has a extension in $V$. If $T$ is in $\mathcal{T}$ and $u$ a finite binary sequence, define

$$
T / u=\{s: u \wedge s \in T\} .
$$

If this set is non-empty, then it is again a member of $\mathcal{T}$ (in which case $T / u$ is the tree of descendants of $u$ ). Elements of $\mathcal{T}$ come with a canonical ordering provided by $<_{\operatorname{lex}}$ and phrases such as the $i$-th element and the minimum element will always refer to the $<_{\text {lex }}$-order in this context. In this paper $\mathbb{N}$ contains 0 and in particular counting will always start at 0 . The letters $i, j, k$, and $l$ will always be used to denote natural numbers.

A tree diagram is a pair $(L, R)$ of elements of $\mathcal{T}$ such that $|L|=|R|$. We view a tree diagram as describing a map of sequences defined by

$$
s_{i} \hat{x} \mapsto t_{i}^{\wedge} x
$$

where $s_{i}$ and $t_{i}$ are the $i$-th elements of $L$ and $R$ respectively and $x$ is any binary sequence. This map is defined not only on all infinite length binary sequences but also on all but finitely many finite binary sequences. The value of the associated map $f$ at a sequence $t$ will be denoted $t \cdot f$ when it is defined. If $t$ is a finite sequence, then we will say that $f$ acts properly on $t$ if $t \cdot f$ is defined and the final digit of $t \cdot f$ agrees with that of $t$. Notice that $f$ acts properly on $t$ unless $s \cdot f$ is undefined for every proper initial part $s$ of $t$. In particular, if $f$ acts properly on $t$, it acts properly on any extension of $t$.

If two tree diagrams define the same map on infinite sequences, then they are said to be equivalent. Every tree diagram is equivalent to a unique minimal tree diagram; 
such a tree diagram is said to be reduced. Furthermore a tree diagram $(S, T)$ is reduced if and only if whenever $i<|S|-1$ it is not the case that both $s_{i}$ and $t_{i}$ end with 0 and $s_{i+1}$ and $t_{i+1}$ end with 1 (where $s_{i}$ and $t_{i}$ are the $i$-th elements of $S$ and $T$ respectively). See [2] for details.

Thompson's group $F$ is the collection of reduced tree diagrams with the operation defined by $f \cdot g=g \circ f$ (i.e., " $f$ followed by $g$ "). $F$ is generated by $\left\{x_{0}, x_{1}\right\}$, where $x_{0}$ and $x_{1}$ are specified by

$$
x_{0}=\left\{\begin{array}{l}
00 \mapsto 0, \\
01 \mapsto 10, \\
1 \mapsto 11,
\end{array} \quad x_{1}=\left\{\begin{array}{l}
0 \mapsto 0, \\
100 \mapsto 10 \\
101 \mapsto 110 \\
11 \mapsto 111
\end{array}\right.\right.
$$

In our discussion of $F$ "generator" will mean an element of the set

$$
\Gamma=\left\{x_{0}, x_{1}, x_{0}^{-1}, x_{1}^{-1}\right\}
$$

(this is really only relevant in Lemma 5.10). This includes the usage of $F \phi l n e r$ in Sections 4 and 5. If $x_{n}$ is defined by $x_{n+1}=x_{0}^{-n} x_{1} x_{0}^{n}$ for $n \geq 1$, then we obtain the generators which yield the infinite presentation of $F$ mentioned in the introduction.

Notice that the existence of constant $C$ satisfying the conclusion of Theorem 1.1 for $\Gamma$ implies the main theorem for all finite generating sets. This is because if $\Gamma^{\prime}$ is any other finite generating set, there is a constant $K>0$ such that any set which is $\varepsilon$-Følner with respect to $\Gamma^{\prime}$ is $K \varepsilon$-Følner with respect to $\Gamma$.

I will also identify elements of $F$ with the corresponding maps on sequences. If $T$ is in $\mathcal{T}$ and $f$ is in $F$ with $f$ defined on all of $T$, then $T \cdot f$ is the pointwise image of $T$ under $f$. It is easily checked that this results in an element of $\mathcal{T}$ and hence this defines a partial right action of $F$ on $\mathcal{T}$. If $T$ is in $\mathcal{T}$ and $f$ is in $F$, then $f$ acts properly on $T$ if it acts properly on the elements of $T$. If $\Gamma$ is a subset of $F$, then $\Gamma$ acts properly on $T$ if each element of $\Gamma$ acts properly on $T$. Observe that if $(S, T)$ is a reduced tree diagram which represents $g$ and $f$ acts properly on $T$, then $(S, T \cdot f)$ is reduced and represents $f \circ g$. If $f$ is in $F$, I will write $\left(L_{f}, R_{f}\right)$ to denote the reduced tree diagram for $f$.

\section{Marginal sets}

In this section we will introduce the notion of a marginal set and collect some basic lemmas which we will use in Sections 4 and 5. Throughout this section "right" in an implicit adjective whenever applicable, although all statements have their corresponding "left" analogs. Fix, for the duration of the section, a group $G$ with a finite symmetric generating set $\Gamma$. 
Definition 3.1. A partial action of $G$ on a set $S$ is a partial function $\cdot: S \times G \rightarrow S$ such that

- $x \cdot e=x$ for all $x \in S$;

- $x \cdot g=y$ if and only if $x=y \cdot g^{-1}$ for all $g \in G$ and $x, y \in S$;

- $x \cdot(g h)=(x \cdot g) \cdot h$ for all $g, h \in G$ and all $x \in S$ for which all computations involving $\cdot$ are defined.

If $E \subseteq S$ and $g$ is in $G$, I will write $E \cdot g$ to denote $\{x \cdot g: x \in E\}$.

Remark 3.2. Since the exponentiation is defined in $G$, it is entirely possible in general for $x \cdot g^{i}$ to be undefined while $x \cdot g^{j}$ is defined for some $i<j$.

Definition 3.3. A weighted $\varepsilon$-F $\phi$ lner set with respect to $\Gamma$ is a function $\mu$ from a finite subset of $S$ into $(0, \infty)$ which satisfies

$$
\sum_{\gamma \in \Gamma} \sum_{s \in S}|\mu(s \cdot \gamma)-\mu(s)|<\varepsilon \sum_{s \in S} \mu(s),
$$

where we adopt with the conventions that $\mu(s)=0$ if $s$ is not in the domain of $\mu$ and $\mu(s \cdot g)=0$ if $s \cdot g$ is undefined. The set $\{s \in S: \mu(s)>0\}$ will be referred to as the support of $\mu$. The function $\mu$ induces a finitely supported measure on $S$, also denoted $\mu$, defined by $\mu(A)=\sum_{s \in A} \mu(s)$. In the remainder of the paper, the generating set will always be clear from the context and we will often suppress mention of it.

Notice that an $\varepsilon$-Følner set is a set $A \subseteq S$ such that the characteristic function $1_{A}$ is a weighted $\varepsilon$-Følner set. I will use $\mu \uparrow A$ to denote $\mu \cdot 1_{A}$. We will need the following property of weighted Følner sets, which is what justifies their added generality in the present paper.

Lemma 3.4. Suppose that $G$ acts partially on sets $S$ and $T$ and that $\mu$ is a weighted $\varepsilon$ Folner set with respect to the action on $S$. If $h: S \rightarrow T$ satisfies that $h(s \cdot \gamma)=h(s) \cdot \gamma$ (with both quantities defined) whenever $\mu(s)+\mu(s \cdot \gamma)>0$ and $\gamma \in \Gamma$, then

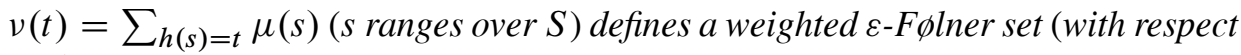
to the action on $T$ ).

Proof. Let $G, S, T, \mu$, and $v$ be as in the statement of the lemma. We need to verify that

$$
\sum_{\gamma \in \Gamma} \sum_{t \in T}|v(t \cdot \gamma)-v(t)|<\varepsilon v(T) .
$$

Let $\gamma \in \Gamma$ and $t \in T$ be fixed for the moment. First observe that if $|v(t \cdot \gamma)-v(t)|>0$, then $t \cdot \gamma$ is defined. This is because otherwise it must be the case that $\nu(t)>0$ and hence there must be an $s$ such that $h(s)=t$ and $\mu(s)>0$. In particular, this implies $t \cdot \gamma$ is defined. 
Next we have that

$$
\sum_{h(u)=t \cdot \gamma} \mu(u)=\sum_{h(u) \cdot \gamma^{-1}=t} \mu(u)=\sum_{h\left(u \cdot \gamma^{-1}\right)=t} \mu(u)=\sum_{h(s)=t} \mu(s \cdot \gamma) .
$$

The first equality is justified by the properties of a partial action; the second equality is justified by our assumption that $\mu(u)>0$ implies $h\left(u \cdot \gamma^{-1}\right)=h(u) \cdot \gamma^{-1}$ with both quantities defined; the third equality is justified by the properties of a partial action and our assumption that $\mu(u)>0$ implies $u \cdot \gamma^{-1}$ is defined. Now it follows that

$$
\begin{aligned}
\sum_{\gamma \in \Gamma} \sum_{t \in T}|v(t \cdot \gamma)-v(t)| & =\sum_{\gamma \in \Gamma} \sum_{t \in T}\left|\sum_{h(s)=t} \mu(s \cdot \gamma)-\mu(s)\right| \\
& \leq \sum_{\gamma \in \Gamma} \sum_{s \in S}|\mu(s \cdot \gamma)-\mu(s)|<\varepsilon \mu(S)=\varepsilon v(T) .
\end{aligned}
$$

Fix a partial action of $G$ on a set $S$ for the duration of this section. If $g$ is in $G$, let $d_{g}$ be the minimum length of a word in $\Gamma$ which evaluates to $g$. We will need the following lemma.

Lemma 3.5. If $\varepsilon>0$, $\mu$ is a weighted $\varepsilon$-Følner set and $g$ is in $G$, then

$$
\sum_{s \in S}|\mu(s \cdot g)-\mu(s)|<2 \varepsilon d_{g} \mu(S) .
$$

Proof. Let $\gamma_{i}\left(i<d_{g}\right)$ be elements of $\Gamma$ such that $g=\prod_{i<d_{g}} \gamma_{i}$. Let $g_{j}=\prod_{i<j} \gamma_{i}$.

$$
\sum_{s \in S}|\mu(s \cdot g)-\mu(s)| \leq \sum_{s \in S} \sum_{i<d_{g}}\left|\mu\left(s \cdot g_{i+1}\right)-\mu\left(s \cdot g_{i}\right)\right| .
$$

Notice that it may be that $s \cdot g_{i+1}$ is defined even though $s \cdot g_{i}$ is not. If this is the case, however, then

$$
\left|\mu\left(s \cdot g_{i+1}\right)-\mu\left(s \cdot g_{i}\right)\right|=\left|\mu\left(\left(s \cdot g_{i+1}\right) \cdot \gamma_{i}^{-1}\right)-\mu\left(s \cdot g_{i+1}\right)\right| .
$$

It follows that

$$
\sum_{i<d_{g}} \sum_{s \in S}\left|\mu\left(s \cdot g_{i+1}\right)-\mu\left(s \cdot g_{i}\right)\right| \leq \sum_{i<d_{g}} \sum_{\sigma= \pm 1} \sum_{s \in S}\left|\mu\left(s \cdot \gamma_{i}^{\sigma}\right)-\mu(s)\right|,
$$

which is less than $2 \varepsilon d_{g} \mu(S)$.

We will be interested in the following notion which ensures that a set has small intersection with any Følner set. The definition is motivated by the following simple observation. If $\mu$ is a finitely additive invariant probability measure on a group $G$ and $E \subseteq G$ satisfies that, for some $g \in G,\left\{E \cdot g^{i}: i \in \mathbb{N}\right\}$ is a pairwise disjoint family, then $\mu(E)=0$. 
Definition 3.6. If $g \in G, I \subseteq S$, and $E \subseteq S$, then $g$ marginalizes $E$ off $I$ if for every $x \in E$ if $x \cdot g^{k} \in E$ and $k>0$, then there is an $i<k$ such that $x \cdot g^{i}$ is in $I$ or is undefined. If $I$ is the empty set, then I will write $g$ marginalizes $E$.

Definition 3.7. The $k$-marginal sets for the partial action of $G$ on $S$ are defined recursively as follows. The empty set is 0-marginal. If there is a decomposition $E=\bigcup_{i<l} E_{i} \subseteq S$ and for each $i<l$, there is a $g_{i} \in G$ and a $k$-marginal set $I_{i}$ such that $g_{i}$ marginalizes $E_{i}$ off $I_{i}$, then $E$ is $(k+1)$-marginal. $E \subseteq S$ is marginal if it is $k$-marginal for some $k<\infty$.

Remark 3.8. For a fixed partial action of a group $G$ on a set $S$, it is immediate from the definition that a finite union of marginal sets is marginal and that a subset of a marginal set is marginal. Additionally, if $E \subseteq S$ and $g$ is in $G$, then $g^{-1}$ marginalizes $E \backslash(E \cdot g)$ off $E$. In particular, if $E$ is marginal, then so is $E \cdot g$.

We will need the following lemma which shows that marginal sets have small intersections with Følner sets.

Lemma 3.9. Suppose that $\varepsilon>0$ and $\mu$ is a weighted $\varepsilon$-Følner set with support $A$. If $E \subseteq S$ and $g$ marginalizes $E$ off $S \backslash A$, then $\mu(E)<2 \varepsilon d_{g} \mu(S)$.

Proof. For each $x \in E \cap A$, let $\Phi(x)$ denote the set of all $x \cdot g^{i}$ such that

- $x \cdot g^{j}$ is defined and in the support of $\mu$ for all $j \leq i$ and

- $\mu\left(x \cdot g^{i+1}\right)<\mu\left(x \cdot g^{j}\right)$ for all $j \leq i$.

Observe that each $\Phi(x)$ is finite and non-empty. Because $E$ is marginalized off $S \backslash A$ by $g,\{\Phi(x): x \in E\}$ is a pairwise disjoint family. Observe that, for a fixed $x \in E$,

$$
\mu(x) \leq \sum_{y \in \Phi(x)} \mu(y)-\mu(y \cdot g)=\sum_{y \in \Phi(x)}|\mu(y \cdot g)-\mu(y)| .
$$

Summing over $x \in E$ and applying Lemma 3.5 now gives the desired estimate.

Lemma 3.10. If $E \subseteq S$ is marginal, then there is a constant $C$ such that if $\varepsilon>0$ and $\mu$ is a weighted $\varepsilon$-Folner set, then $\mu(E)<C \varepsilon \mu(S)$.

Proof. I will prove by induction on $k$ that if $E$ is $k$-marginal, then the conclusion of the lemma holds. If $k=0$, then $E$ is in fact empty and $\mu(E)=0$. Next suppose that $E$ is $(k+1)$-marginal. Let $I_{i}(i<l), g_{i}(i<l)$, and $E_{i}(i<l)$ be such that

- for each $i<l, g_{i}$ is in $G$ and marginalizes $E_{i}$ off $I_{i}$;

- for each $i<l, I_{i}$ is $k$-marginal;

- $E=\bigcup_{i<l} E_{i}$. 
By our inductive assumption, there are $C_{i}(i<l)$ such that if $\mu$ is a weighted $\varepsilon$ Følner set, then $\mu\left(I_{i}\right)<C_{i} \varepsilon \mu(S)$. Set $C=\sum_{i<l} C_{i}+2 d_{g_{i}}$. Now if $\mu$ is a weighted $\varepsilon$-Følner set, then by applying Lemma 3.9 to the restriction of $\mu$ to $S \backslash \bigcup_{i \in I} I_{i}$ we obtain

$$
\mu(E)=\mu\left(\bigcup_{i<l} I_{i}\right)+\mu\left(\bigcup_{i<l} E_{i} \backslash \bigcup_{i<l} I_{i}\right) \leq \sum_{i<l} C_{i} \varepsilon \mu(S)+2 d_{g_{i}} \varepsilon \mu(S)=C \varepsilon \mu(S) .
$$

Lemma 3.11. If $\mu$ is a weighted $\varepsilon$-Følner set and $v$ is a function from a finite subset of $S$ into $[0, \infty)$ with $v \leq \mu$ pointwise and $v(S) \geq(1-\delta) \mu(S)$ for some $\delta$ satisfying $0<\delta<1$, then $v$ is a weighted $(\varepsilon+2|\Gamma| \delta) /(1-\delta)$-Følner set.

Proof. Let $\mu$ and $v$ be given as in the statement of the lemma. Fix an $s \in S$ and $\gamma \in \Gamma$. If $v(s \cdot \gamma) \geq v(s)$, then

$$
|v(s \cdot \gamma)-v(s)| \leq \mu(s \cdot \gamma)-\mu(s)+\mu(s)-v(s) .
$$

If $v(s \cdot \gamma) \leq v(s)$, then

$$
|v(s \cdot \gamma)-v(s)| \leq \mu(s)-\mu(s \cdot \gamma)+\mu(s \cdot \gamma)-v(s \cdot \gamma) .
$$

In either case, we have

$$
|v(s \cdot \gamma)-v(s)| \leq|\mu(s \cdot \gamma)-\mu(s)|+\mu(s)-v(s)+\mu(s \cdot \gamma)-v(s \cdot \gamma) .
$$

Summing over $s \in S$ and $\gamma \in \Gamma$ and combining this with our hypotheses we obtain:

$$
\sum_{\gamma \in \Gamma} \sum_{s \in S}|v(s \cdot \gamma)-v(s)|<\varepsilon \mu(S)+2|\Gamma| \delta \mu(S) \leq \frac{\varepsilon+2|\Gamma| \delta}{1-\delta} v(S) .
$$

Lemma 3.12. If $E \subseteq S$ is marginal and $S \backslash E$ is non-empty, then there is a constant $C$ such that if $\mu$ is a weighted $\varepsilon-F \phi$ lner set and $C \varepsilon \leq 1$, then $\mu \uparrow(S \backslash E)$ is a weighted $C \varepsilon$-Følner (and in particular the support of $\mu \uparrow(S \backslash E)$ is non-empty).

Proof. Let $E$ be marginal and let $C_{0}$ be such that if $\varepsilon>0$ and $\mu$ is a weighted $\varepsilon$-Følner set, then $\mu(E)<C_{0} \varepsilon \mu(S)$ and hence $\mu(S \backslash E)>\left(1-C_{0} \epsilon\right) \mu(S)$. Set $C=2\left(1+2 C_{0}|\Gamma|\right)$ and suppose that $\varepsilon>0$ satisfies $C \varepsilon \leq 1$ and that $\mu$ is a weighted $\varepsilon$-Følner set. It follows that $C_{0} \varepsilon \leq 1 / 2$ and hence

$$
\frac{\varepsilon+2|\Gamma| C_{0} \varepsilon}{1-C_{0} \varepsilon} \leq 2\left(1+2|\Gamma| C_{0}\right) \varepsilon
$$

Observe that the support of $\mu$ is not contained in $E$ since $\mu(E)<C_{0} \varepsilon \mu(S)<\mu(S)$. Hence by applying Lemma 3.11 with $\delta=C_{0} \varepsilon$ and $v=\mu \uparrow(S \backslash E)$, we obtain that $\mu \uparrow(S \backslash E)$ is $C \varepsilon$-Følner. 
Definition 3.13. A subset $A \subseteq G$ is $\Gamma$-connected if whenever $x$ and $y$ are in $A$, there are $\gamma_{i}(i<l)$ in $\Gamma$ such that, setting $x_{0}=x$ and $x_{i+1}=x_{i} \cdot \gamma_{i}$, then $x_{i}$ is defined for each $i \leq l$ and $y=x_{l}$. A maximal $\Gamma$-connected subset of a given $B \subseteq G$ is said to be a $\Gamma$-connected component of $B$.

Lemma 3.14. If $\varepsilon>0$ and $\mu$ is a weighted $\varepsilon$-Følner set, then the support of $\mu$ contains a $\Gamma$-connected component $A$ such that $\mu \uparrow A$ is $\varepsilon$-Følner.

Proof. Let $A_{i}(i<l)$ enumerate the $\Gamma$-connected components of the support of $\mu$. Then since

$$
\sum_{i<l} \sum_{\gamma \in \Gamma} \sum_{s \in A_{i}}|\mu(s \cdot \gamma)-\mu(s)|<\varepsilon \sum_{i<l} \mu\left(A_{i}\right),
$$

there must exist an $i<l$ such that

$$
\sum_{\gamma \in \Gamma} \sum_{s \in A_{i}}|\mu(s \cdot \gamma)-\mu(s)|<\varepsilon \mu\left(A_{i}\right) .
$$

Since $A_{i}$ is a $\Gamma$-connected component, $\mu(s \cdot \gamma)=\mu \uparrow A_{i}(s \cdot \gamma)$ whenever $s$ is in $A_{i}$ and therefore $A_{i}$ is $\varepsilon$-Følner.

This lemma has the following useful consequence.

Lemma 3.15. Let $G$ be a group with a finite generating set $\Gamma$, acting on itself from the right. If $A \subseteq G$ is a $\varepsilon$-F $\phi$ lner set, then there is a $B \subseteq G$ which is an $\varepsilon$-Folner set such that $B$ is $\Gamma$-connected, $B$ contains the identity, and $|B| \leq|A|$.

Proof. Fix an $\varepsilon$-Følner set $A$ and let $C$ be a $\Gamma$-connected component of $A$ which is $\varepsilon$-Følner. Let $g$ be any element of $C$ and define $B=g^{-1} C$. It is easily verified that $C$ is still $\Gamma$-connected and (right) $\varepsilon$-Følner.

\section{Følner sets of trees}

Rather than studying Følner sets in $F$ directly, it will be easier to deal with weighted Følner sets in the partial right action of $F$ on $\mathcal{T}$ which I will refer to as weighted Følner sets of trees. These are essentially weighted right Følner sets consisting of positive elements of $F$ (positive with respect to the infinite presentation mentioned above). The reformulation of the amenability problem for $F$ in terms of the existence of Følner sets of positive elements is well known [6] and part of a more general phenomenon (see [8], 1.28), but we will need the more precise analytic consequences of the following lemmas.

Lemma 4.1. For every finite binary sequence $u$, the set $E_{u}$ of all $f \in F$ such that $u$ is not extended by an element of $R_{f}$ is marginal with respect to the right action of $F$ on itself. 
Proof. Let $u$ be fixed and let $g$ be the element of $F$ which is the identity on sequences which do not extend $u$ and which satisfies

$$
\left(u^{\wedge} v\right) \cdot g=u^{\wedge}\left(v \cdot x_{0}\right)
$$

for all sequences $v$ for which $v \cdot x_{0}$ is defined.

Now let $f$ be an element of $E_{u}$ and let $t$ be the initial part of $u$ which is in $R_{f}$ (such a $t$ exists by our assumption that $u$ does not have an extension in $R_{f}$ ). Let $s=t \cdot f^{-1}$. That is, $s$ is the element of $L_{f}$ such that

$$
\left|\left\{a \in L_{f}: a<_{\operatorname{lex}} s\right\}\right|=\left|\left\{b \in R_{f}: b<_{\operatorname{lex}} t\right\}\right| .
$$

Observe that $f \cdot g^{n}$ can be represented by the tree diagram $(A, B)$ defined by:

$$
\begin{aligned}
& A=\left(L_{f} \backslash\{s\}\right) \cup\left\{s^{\wedge} v: s^{\wedge} v \in L_{g^{n}}\right\}, \\
& B=\left(R_{f} \backslash\{t\}\right) \cup\left\{t^{\wedge} v: t^{\wedge} v \in R_{g^{n}}\right\} .
\end{aligned}
$$

That $(A, B)$ is a reduced tree diagram follows from the characterization mentioned in Section 2 and the following facts:

- $\left(L_{f}, R_{f}\right)$ and $\left(L_{g^{n}}, R_{g^{n}}\right)$ are reduced;

- $\left|\left\{a \in A: a<_{\operatorname{lex}} s\right\}\right|=\left|\left\{b \in B: b<_{\operatorname{lex}} t\right\}\right|$;

- the minimum (maximum) elements of the sets

$$
\left\{s^{\wedge} v: t^{\wedge} v \in L_{g^{n}}\right\} \quad \text { and } \quad\left\{t^{\wedge} v: t^{\wedge} v \in R_{g^{n}}\right\}
$$

end in 0 (respectively 1 ).

Consequently $u$ is extended by an element of $R_{f \cdot g^{n}}$ for all $n>0$ and hence $g$ marginalizes $E_{u}$.

Lemma 4.2. There is a constant $C$ such that if $A \subseteq F$ is a (right) $\varepsilon$-Følner set, then there is a weighted $C \varepsilon$-Følner set of trees supported on a subset of $\left\{R_{f}: f \in A\right\}$.

Proof. Let $U$ consist of all binary sequences of length 4, noting that if $T$ dominates $U$, then every element of $\Gamma$ acts properly on $T$. By Lemma 4.1, the set of $f \in F$ such that $U$ is not dominated by $R_{f}$ is marginal. By Lemma 3.12, there is a constant $C>1$ such that if $A$ is $\varepsilon$-Følner and

$$
A_{0}=\left\{f \in A: U \text { is dominated by } R_{f}\right\},
$$

then $A_{0}$ is $C \varepsilon$-Følner. Observe that if $\gamma$ is a generator and $f$ is in $A_{0}$, then $L_{f \cdot \gamma}=L_{f}$ and $R_{(f \cdot \gamma)}=\left(R_{f}\right) \cdot \gamma$. We are now finished by Lemma 3.4 applied to $h(f)=R_{f}$ and $\mu=1_{A_{0}}$. 


\section{An operation on elements of $\mathcal{T}$}

In this section I will define an operation $\partial$ on elements of $\mathcal{T}$ which reduces their size logarithmically.

If $T$ is in $\mathcal{T}$, then the end points of $T$ are the maximum and minimum elements of $T$. All other elements of $T$ are said to be interior.

Definition 5.1. Suppose that $T$ is in $\mathcal{T}$. $\partial T$ is the maximum $U \in \mathcal{T}$ (with respect to the order of domination) which is dominated by $T$ which satisfies the following defining conditions:

(1) $U$ contains extensions of both 01 and 10;

(2) one of the following holds:

- if $u<_{\operatorname{lex}} v$ are interior elements of $U$, then $2|T / u| \leq|T / v|$;

- if $u<_{\operatorname{lex}} v$ are interior elements of $U$, then $2|T / v| \leq|T / u|$;

(3) the minimum (respectively the maximum) interior element of $U$ terminates with a 1 (respectively with a 0 ).

If no such $U$ exists, then $\partial T$ is defined to be the trivial tree.

The following lemma justifies the use of maximum in the definition of $\partial T$.

Lemma 5.2. If there is a $U$ satisfying the defining conditions for $\partial T$, then there is a maximum such $U$ with respect to the order of domination.

Remark 5.3. Condition (3) is necessary to ensure the uniqueness of maximal elements of $\mathcal{T}$ which satisfying the defining conditions for $\partial T$.

Proof. Suppose for contradiction that $U$ and $V$ are distinct maximal elements of $\mathcal{T}$, each dominated by $T$, which satisfy the defining conditions of $\partial T$. First I claim that condition (2) is satisfied in the same way for $U$ and $V$. For this, it is sufficient to show that if $W$ is in $\mathcal{T}$, contains extensions of both 01 and 10 , and satisfies the first (second) option of condition (2) for $T$, then $|T / 01|<|T / 10|$ (respectively $|T / 10|<|T / 01|)$. Suppose that $W$ satisfies the first option (the other case is symmetric). Let $w$ be the greatest element of $W$ extending 01 and $w^{\prime}$ be the least element of $W$ extending 10. Condition (2) implies that if $u_{i}(i \leq l)$ are the elements of $W$ which extend 01 , then

$$
\sum_{i<l}\left|T / u_{i}\right|<\left|T / u_{l}\right|=|T / w|
$$

and hence $|T / 01|<2|T / w|$. It follows that

$$
|T / 01|<2|T / w| \leq\left|T / w^{\prime}\right| \leq|T / 10| .
$$


By replacing $U$ and $V$ with their mirror images if necessary, we will assume that the quantity $|T / u|$ is increasing as $u$ increases in $U$ (or equivalently as $u$ increases in $V$ ). Since $U \triangle V$ is non-empty, there is a minimum element of $U$ which either properly extends an element of $V$ or is properly extended by an element of $V$. By exchanging the roles of $U$ with $V$ if necessary, we may assume the former occurs.

First suppose that the minimum elements of $U$ and $V$ are the same. Let $u$ be the greatest element of $U$ such that it and all of its $<_{\operatorname{lex}}$-predecessors extend an element of $V$ (this extension may not be proper in the case of the predecessors of $u$ ). Let $v$ be the element of $V$ which $u$ extends. Define

$$
W=\left\{w \in U: w \leq_{\operatorname{lex}} u\right\} \cup\left\{w \in V: v<_{\operatorname{lex}} w\right\}
$$

and observe that $W$ is in $\mathcal{T}$. Notice that $|T / u| \leq|T / v|$. Also observe that since $U$ does not dominate $V, u$ is not the maximum element of $U$ and therefore if $x$ is an element of $U$ such that $x \leq_{\operatorname{lex}} u$, then $|T / x| \leq|T / u|$. It follows that if $x$ is an interior element of $W$ in $\left\{w \in U: w \leq_{\operatorname{lex}} u\right\}$ and $y$ is an interior element of $W$ in $\left\{w \in V: v<_{\operatorname{lex}} w\right\}$, then $x \leq_{\operatorname{lex}} u$ and $v<_{\operatorname{lex}} y$ which in turn implies

$$
2|T / x| \leq 2|T / u| \leq 2|T / v| \leq|T / y| .
$$

Since both $U$ and $V$ satisfy condition (2), if $x<_{\operatorname{lex}} y$ are interior elements of $W$ and either both are in $U$ or both are in $V$, then $2|T / x| \leq|T / y|$. It follows that $W$ satisfies condition (2) as well. Since $U$ and $V$ have the same minimum element and both satisfy condition (3), $U$ and $V$ have the same minimal interior element. Thus the minimum interior element of $U$ is also the minimum interior element of $W$. Also, since $V$ is not dominated by $U, u$ is not the maximum element of $W$ and $v$ is not the maximum element of $V$. Moreover, it can not be the case that $v$ is the maximum interior element of $V$. If this were the case, then $v=x^{\wedge} 0$ for some $x$. It would then follow that $x^{\wedge} 1$ would be the maximum element of $V$ and would have an extension in $U$. This would imply that $U$ dominates $V$, which we assumed was not the case. It follows that the maximum interior element of $W$ is the same as the maximum interior element of $V$. Therefore $W$ satisfies condition (3). But now $W$ satisfies the defining conditions for $\partial T$, contradicting the maximality of $V$.

Now suppose that the minimum elements of $U$ and $V$ differ. Let $v$ be such that $v^{\wedge} 0$ is the minimum element of $V$, noting that $v^{\wedge} 1$ is the minimum interior element of $V$. Define

$$
W=\left\{w \in U: v^{\wedge} 0 \subseteq w\right\} \cup\left\{w \in V: v^{\wedge} 0<_{\operatorname{lex}} w\right\}
$$

and observe that $W$ is in $\mathcal{T}$. Since $v^{\wedge} 0$ is not in $U$, it must be that both $v^{\wedge} 00$ and $v^{\wedge} 01$ have extensions in $U$ and in particular, the minimum interior element of $W$ is the same as the one of $U$. Since $V$ is not dominated by $U, v \neq\langle\rangle$. Since $v^{\wedge} 0$ is the minimum element of $V$, the entries of $v$ are all 0 and hence $v^{\wedge} 1$ is not the maximum element of $V$ or of $W$. Since every element of $W$ not extending $v^{\wedge} 0$ is in $V$, it follows 
that the maximum interior element of $W$ is the same as the maximum interior element of $V$. Therefore $W$ satisfies condition (3). Next suppose that $x<_{\operatorname{lex}} y$ are interior elements of $W$. If both $x$ and $y$ are in $U$ or both are in $V$, then $2|T / x| \leq|T / y|$ follows from the fact that $U$ and $V$ satisfy condition (2) and that the minimum interior element of $W$ is the same as that of $U$ and that the maximum interior element of $W$ is the same as that of $V$. Next suppose that $x$ is in $\left\{w \in U: v^{\wedge} 0 \subseteq w\right\}$ and $y$ is in $\left\{w \in V: v^{\wedge} 0<_{\operatorname{lex}} w\right\}$. Observe that either $y=v^{\wedge} 1$ or $v^{\wedge} 1<_{\operatorname{lex}} y$. Since $v^{\wedge} 0$ is extended by an element of $U$, it must be that $v^{\wedge} 1$ is also extended by an element $w$ of $U$ which is in the interior of $U$. We now have

$$
2|T / x| \leq|T / w| \leq\left|T / v^{\wedge} 1\right| \leq|T / y|
$$

(This is where the crucial use of condition (3) occurs.) Thus $W$ satisfies condition (2). Again, $W$ satisfies the defining conditions for $\partial T$, contradicting the maximality of $V$.

Lemma 5.4. If $\partial T$ has $n$ elements, then $T$ has more than $2^{n-2}$ elements.

Proof. There are $n-2$ interior elements of $\partial T$ and thus by condition (2) the total number of elements of $T$ which extend an interior element of $\partial T$ is at least $\sum_{i=1}^{n-2} 2^{i-1}=2^{n-2}-1$. Since $\partial T$ is dominated by $T$, there are at least two elements of $T$ remaining to be counted, putting the total greater than $2^{n-2}$.

Lemma 5.5. If $g$ is in $F, T$ is in $\mathcal{T}$, and $g$ acts properly on $\partial T$, then $\partial(T \cdot g)=(\partial T) \cdot g$.

Proof. First I will verify that if $g$ and $T$ are as in the statement of the lemma, then $(\partial T) \cdot g$ is dominated by $\partial(T \cdot g)$. Since the action of $g$ on the elements of $\partial T$ does not change their final digit, $(\partial T) \cdot g$ satisfies condition (3). Since the action of $g$ preserves lexicographic order and extension of sequences, $(\partial T) \cdot g$ satisfies condition (2). Finally, since the action of $g$ on $\partial T$ is proper, $\partial T \cdot g$ satisfies condition (1). It follows that $(\partial T) \cdot g$ is dominated by $\partial(T \cdot g)$.

Next observe that if $g$ acts properly on $T$, then $g^{-1}$ acts properly on $T \cdot g$. It follows that $\partial(T \cdot g) \cdot g^{-1}$ is dominated by $\partial\left(T \cdot g \cdot g^{-1}\right)=\partial T$. Since $g$ and $g^{-1}$ are injections, it follows that $\partial(T \cdot g)$ and $(\partial T) \cdot g$ have the same elements and hence are equal.

Definition 5.6. Let $\mathcal{E}$ be the set of all $T \in \mathcal{T}$ such that neither of the following inequalities hold:

$$
\begin{array}{ll}
\text { (+) } & |T / 001|<|T / 01|<|T / 10|, \\
\text { (-) } & |T / 001|>|T / 01|>|T / 10| .
\end{array}
$$

Define

$$
\begin{aligned}
& \mathcal{T}^{+}=\{T \in \mathcal{T}:|T / 001|<|T / 01|<|T / 10|\} \\
& \mathcal{T}^{-}=\{T \in \mathcal{T}:|T / 001|>|T / 01|>|T / 10|\} .
\end{aligned}
$$


Lemma 5.7. $\mathcal{E}$ is marginal.

Proof. Define the following elements of $F$ :

$$
a=\left\{\begin{array}{l}
000 \mapsto 000, \\
0010 \mapsto 001, \\
0011 \mapsto 0100, \\
01 \mapsto 0101, \\
100 \mapsto 011, \\
101 \mapsto 10, \\
11 \mapsto 11,
\end{array} \quad b=\left\{\begin{array}{l}
000 \mapsto 000, \\
0010 \mapsto 001, \\
0011 \mapsto 01, \\
01 \mapsto 100, \\
10 \mapsto 101, \\
11 \mapsto 11,
\end{array}\right.\right.
$$

(i.e., $a=x_{0}^{2} x_{1} x_{4} x_{2}^{-2} x_{0}^{-2}$ and $b=x_{0}^{2} x_{1} x_{3}^{-1} x_{0}^{-2}$ ). Define

$$
\begin{aligned}
& \mathcal{E}_{a}=\{T \in \mathcal{T}: \max (|T / 001|,|T / 10|)=|T / 01|\}, \\
& \mathcal{E}_{b}=\{T \in \mathcal{T}: \max (|T / 001|,|T / 10|)<|T / 01|\} .
\end{aligned}
$$

Notice that $T$ is in $\mathcal{E}_{\max }=\varepsilon_{a} \cup \mathcal{E}_{b}$ if and only if

$$
|T / 01|=\max (|T / 001|,|T / 01|,|T / 10|) .
$$

Furthermore, if $T$ is in $\mathscr{E} \backslash \mathcal{E}_{\max }$, then

$$
|T / 01|=\min (|T / 001|,|T / 01|,|T / 10|) .
$$

Observe that for all $T \in \mathcal{T}$ such that $T \cdot a$ is defined, we have

$$
\begin{aligned}
|(T \cdot a) / 001| & =|T / 0010|<|T / 001|, \\
|(T \cdot a) / 01| & =|T / 0011|+|T / 01|+|T / 100|>|T / 01|, \\
|(T \cdot a) / 10| & =|T / 101|<|T / 10| .
\end{aligned}
$$

Therefore if $T \cdot a$ is in $\left(\mathcal{E}_{a} \cup \mathcal{E}_{b}\right) \cdot a$, then

$$
\max (|(T \cdot a) / 001|,|(T \cdot a) / 10|)<|(T \cdot a) / 01|
$$

and hence $T \cdot a$ is in $\varepsilon_{b}$. Since $\varepsilon_{a}$ is disjoint from $\mathcal{E}_{b}$, this shows that $a$ marginalizes $\varepsilon_{a}$.

Observe that for all $T \in \mathcal{T}$ such that $T \cdot b$ is defined, we have

$$
\begin{aligned}
|(T \cdot b) / 001| & =|T / 0010|<|T / 001|, \\
|(T \cdot b) / 01| & =|T / 0011|<|T / 001|, \\
|(T \cdot b) / 10| & =|T / 01|+|T / 10|>|T / 10| .
\end{aligned}
$$

Define

$$
\mathcal{R}=\{T \in \mathcal{T}: \max (|T / 001|,|T / 01|)<|T / 10|\} .
$$


The above inequalities show that $\left(\varepsilon_{b} \cup \mathcal{R}\right) \cdot b \subseteq \mathcal{R}$. Since $\varepsilon_{b}$ is disjoint from $\mathcal{R}$, this shows that $b$ marginalizes $\varepsilon_{b}$.

Clearly the elements of $\mathcal{E} \backslash \mathcal{E}_{\max }$ lie in one of the following sets:

$$
\begin{aligned}
& \mathcal{E}_{1}=\{T \in \mathcal{T}:|T / 001|>|T / 01|=|T / 10|\}, \\
& \mathcal{E}_{2}=\{T \in \mathcal{T}:|T / 01|<\min (|T / 001|,|T / 10|)\}, \\
& \mathcal{E}_{3}=\{T \in \mathcal{T}:|T / 001|=|T / 01|<|T / 10|\} .
\end{aligned}
$$

The proof will therefore be complete once it has been show that $x_{0}$ marginalizes $\varepsilon_{1} \cup \mathcal{E}_{2}$ off $\mathcal{E}_{\max }$ and that $x_{0}$ marginalizes $\mathcal{E}_{3}$ off $\mathcal{E}_{\max }$.

If $T$ is in $\mathcal{T}$, then

$$
\begin{aligned}
& \left|\left(T \cdot x_{0}\right) / 01\right|=|T / 001|, \\
& \left|\left(T \cdot x_{0}\right) / 10\right|=|T / 01| .
\end{aligned}
$$

If $T$ is in $\mathcal{T}^{-} \cup \mathcal{E}_{1} \cup \mathcal{E}_{2}$, then $|T / 001|>|T / 01|$ and thus

$$
\left|\left(T \cdot x_{0}\right) / 01\right|>\left|\left(T \cdot x_{0}\right) / 10\right| \text {. }
$$

This yields

$$
\left(\mathcal{T}^{-} \cup \mathcal{E}_{1} \cup \mathcal{E}_{2}\right) \cdot x_{0} \subseteq \mathcal{T}^{-} \cup \mathcal{E}_{\max }
$$

If $T$ is in $\varepsilon_{3}$, then $|T / 001|=|T / 01|$ and thus

$$
\left|\left(T \cdot x_{0}\right) / 01\right|=\left|\left(T \cdot x_{0}\right) / 10\right| \text {. }
$$

This yields

$$
\varepsilon_{3} \cdot x_{0} \subseteq \varepsilon_{1} \cup \varepsilon_{\max } .
$$

This shows that $x_{0}$ marginalizes $\mathcal{E}_{1} \cup \mathcal{E}_{3}$ off $\mathcal{E}_{\max }$ and that $x_{0}$ marginalizes $\mathcal{E}_{3}$ off $\varepsilon_{\text {max }}$.

Definition 5.8. Let $\mathcal{E}^{*}$ be the set of all $T \in \mathcal{T}$ such that neither of the following inequalities hold:

$$
\begin{array}{ll}
(2 \times) & 2|T / 001| \leq|T / 01| \leq \frac{1}{2}|T / 10|, \\
\left(\frac{1}{2} \times\right) & \frac{1}{2}|T / 001| \geq|T / 01| \geq 2|T / 10| .
\end{array}
$$

Observe that if $T$ is not in $\mathcal{E}^{*}$ and $T$ contains extensions of both 01 and 10 , then $\partial T$ is not the trivial tree since $\{00,01,10,11\}$ is then an element of $\mathcal{T}$ which satisfies the defining conditions for $\partial T$.

Lemma 5.9. If $T$ satisfies $(+)$ and $\gamma$ is a generator, then either $T \cdot \gamma$ is undefined, $T \cdot \gamma$ is in $\mathcal{E}$, or else $T \cdot \gamma$ satisfies $(+)$ (and similarly for $(-)$ ). In particular, if $\mathcal{A} \subseteq \mathcal{T} \backslash \mathcal{E}^{*}$ is $\Gamma$-connected, then either all elements of $\mathcal{A}$ satisfy $(2 \times)$ or all elements of $\mathcal{A}$ satisfy $\left(\frac{1}{2} \times\right)$. 
Proof. This follows from the following equalities which hold whenever the relevant action is defined:

$$
\begin{aligned}
T / 001 & =\left(T \cdot x_{0}\right) / 01, \\
T / 01 & =\left(T \cdot x_{0}\right) / 10, \\
T / 001 & =\left(T \cdot x_{1}^{ \pm}\right) / 001, \\
T / 01 & =\left(T \cdot x_{1}^{ \pm}\right) / 01 .
\end{aligned}
$$

Lemma 5.10. $\mathcal{E}^{*}$ is marginal.

Remark 5.11. Lemmas 5.9 and 5.10 have non trivial qualitative consequences for $F$-invariant probability measures on $\mathcal{T}$. If $\mu$ is an $F$-invariant probability measure on $\mathcal{T}$, then $\mu\left(\mathcal{E}^{*}\right)=0$. Furthermore, $\mu\left(\mathcal{T}^{+} \Delta\left(\mathcal{T}^{+} \cdot \Gamma\right)\right)=0$ and therefore if $\mu$ is additionally ergodic, then it must be that $\mu$ assigns measure 1 either to the set of elements of $\mathcal{T}$ which satisfy $(2 \times)$ or else to the set of those which satisfy $\left(\frac{1}{2} \times\right)$.

Proof. Define the following elements of $F$ :

$$
c=\left\{\begin{array}{l}
00 \mapsto 0, \\
01 \mapsto 100, \\
10 \mapsto 101, \\
11 \mapsto 11,
\end{array} \quad d=\left\{\begin{array}{l}
000 \mapsto 00, \\
001 \mapsto 010 \\
01 \mapsto 011, \\
1 \mapsto 1,
\end{array}\right.\right.
$$

(i.e., $c=x_{0} x_{1}^{-1}$ and $\left.d=x_{0}^{2} x_{1}^{-1} x_{0}^{-1}\right)$. Define

$$
\begin{aligned}
& \mathcal{X}=\left\{T \in \mathcal{T}^{+}: 2|T / 01| \leq|T / 10|\right\} \\
& \mathcal{E}_{4}=\left\{T \in \mathcal{T}^{+}: 2|T / 01|>|T / 10|\right\} \\
& \mathcal{E}_{5}=\left\{T \in \mathcal{T}^{+}: 2|T / 001|>|T / 01|\right\}
\end{aligned}
$$

I first claim that $\left(\mathcal{X} \cup \mathcal{E}_{4}\right) \cdot c \subseteq \mathcal{X} \cup \mathcal{E}$. To see this, suppose that $T$ is in $\mathcal{T}^{+}$. Then

$$
2|(T \cdot c) / 01|=2|T / 001|<|T / 01|+|T / 10|=|(T \cdot c) / 10| .
$$

and hence if $T \cdot c$ is in $\mathcal{T}^{+}$, it is in $\mathcal{X}$. Since $T \cdot c$ is not in $\mathcal{T}^{-}$, it is either in $\mathcal{T}^{+}$or in $\mathcal{E}$. This proves the claim. Since $\mathcal{E}_{4}$ is disjoint from $\mathcal{X}$, it follows that $c$ marginalizes $\varepsilon_{4}$ off $\mathcal{E}$ and hence that $\varepsilon_{4}$ is marginal according to Lemma 5.7. Also, by Lemma 5.9, $\mathcal{E}_{5} \cdot x_{0} \subseteq \mathcal{E}_{4} \cup \mathcal{E}$ and therefore $\mathcal{E}_{5}$ is marginal because $x_{0}$ marginalizes $\mathcal{E}_{5}$ off $\mathcal{E}_{4} \cup \mathcal{E}$.

Next define

$$
\begin{aligned}
y & =\left\{T \in \mathcal{T}^{-}:|T / 01| \geq 2|T / 10|\right\}, \\
\mathcal{E}_{6} & =\left\{T \in \mathcal{T}^{-}:|T / 01|<2|T / 10|\right\}, \\
\mathcal{E}_{7} & =\left\{T \in \mathcal{T}^{-}:|T / 001|<2|T / 01|\right\} .
\end{aligned}
$$


Arguing as above, $\left(\mathcal{Y} \cup \mathcal{E}_{6}\right) \cdot d \subseteq \mathcal{Y} \cup \mathcal{E}$ and hence $d$ marginalizes $\mathcal{E}_{6}$ off $\mathcal{E}$. Also $\varepsilon_{7} \cdot x_{0} \subseteq \varepsilon_{6} \cup \mathcal{E}$ and consequently both $\varepsilon_{6}$ and $\mathcal{E}_{7}$ are marginal. Since $\mathcal{E}^{*}=\mathscr{E} \cup \bigcup_{i=4}^{7} \varepsilon_{i}, \mathcal{E}^{*}$ is marginal and the proof is complete.

Lemma 5.12. The set

$$
\{T \in \mathcal{T}: \Gamma \text { does not act properly on } \partial T\}
$$

is marginal.

Proof. First observe that

$$
1^{i+1} 0 \cdot x_{0}^{-i}=10, \quad 1^{i} 0 \cdot x_{0}^{-i}=01 .
$$

Hence if $T$ is in $\mathcal{T}$ and $T \cdot x_{0}^{-i}$ is defined, then

$$
\begin{aligned}
\left|T / 1^{i} 0\right| & =\left|\left(T \cdot x_{0}^{-i}\right) / 01\right|, \\
\left|T / 1^{i+1} 0\right| & =\left|\left(T \cdot x_{0}^{-i}\right) / 10\right| .
\end{aligned}
$$

By Lemma 5.10, $\mathcal{E}^{*}$ is marginal. Also, it follows immediately from the definitions that for each $f$ in $F$, the set of all $T \in \mathcal{T}$ for which $T \cdot f$ is undefined is marginalized off the empty set by $f$. By Remark 3.8, it follows that

$$
\mathcal{E}^{* *}=\bigcup_{i=0}^{16} \mathcal{E}^{*} \cdot x_{0}^{i} \cup\left\{T \in \mathcal{T}: \exists i \leq 16\left(T \cdot x_{0}^{-i} \text { is undefined }\right)\right\}
$$

is marginal as well. Observe that if $T$ is not in $\mathcal{E}^{* *}$, then $\left\{T \cdot x_{0}^{-i}: 0 \leq i \leq 16\right\}$ is a $\Gamma$-connected subset of $\mathcal{T} \backslash \mathcal{E}^{*}$ and therefore by Lemma 5.9, one of the following two assertions holds:

$$
\begin{aligned}
2\left|T / 1^{i} 0\right| & <\left|T / 1^{i+1} 0\right| \text { for all } i<16, \\
2\left|T / 1^{i+1} 0\right| & <\left|T / 1^{i} 0\right| \text { for all } i<16 .
\end{aligned}
$$

That is, the set of all $T$ which satisfy neither of these assertions is marginal.

Now let $U$ consist of all binary sequences of length 4, noting that $U$ satisfies conditions (1) and (3). Furthermore if $T$ is an element of $\mathcal{T}$ such that $\partial T$ dominates $U$, then every element of $\Gamma$ acts properly on $\partial T$. Set $R=\left\{1^{i} 0: i<14\right\} \cup\left\{1^{15}\right\}$ and let $g$ denote the element of $F$ defined by the tree diagram $(U, R)$. If $U$ fails to satisfy condition (2) with respect to $T$, then $U \cdot g$ fails to satisfy condition (2) with respect to $T \cdot g$ and, in particular, $T \cdot g$ must be in $\mathcal{E}^{* *}$. Therefore the set of $T$ such that $U$ does not satisfy the defining conditions for $\partial T$ is marginalized by $g$ off $\varepsilon^{* *}$.

Lemma 5.13. There is a constant $C$ such that if $\mu$ is a weighted $\varepsilon$-F $\phi$ lner set of trees and $C \varepsilon \leq 1$, then there is a weighted $C \varepsilon$-Folner set of trees which is supported on a subset of

$\{\partial T:(\mu(T)>0) \wedge(\partial T$ is non trivial $) \wedge(\Gamma$ acts properly on $\partial T)\}$. 
Proof. By Lemmas 3.12 and 5.12, there is a $C$ such that if $\mu$ is a weighted $\varepsilon$-Følner set of trees and

$$
\mathcal{A}=\{T \in \mathcal{T}:(\mu(T)>0) \wedge(\Gamma \text { acts properly on } \partial T)\},
$$

then $\mu \uparrow \mathcal{A}$ is $C \varepsilon$-Følner. Now let such a $\mu$ be given and define $\mathcal{A}$ as above. By Lemma 5.5,

$$
(\partial T) \cdot \gamma=\partial(T \cdot \gamma)
$$

whenever $\gamma$ is a generator and $T$ is in $\mathcal{A}$. Applying Lemma 3.4 to $\mu \uparrow \mathcal{A}$ and $h=\partial$ gives the desired conclusion.

Now we are ready to complete the proof of Theorem 1.1. I will first prove the following claim.

Claim 5.14. There exists a constant $K>1$ such that if $A \subseteq F$ is a $K^{-n}$-Følner set, then $A$ contains an element with a tree diagram whose trees each contain at least $\exp _{n}(0)$ elements.

Proof. By Lemmas 4.2 and 5.13 there is a constant $K>1$ such that

(1) if $A \subseteq F$ is an $\varepsilon$-Følner set and $K \varepsilon \leq 1$, then there is a weighted $K \varepsilon$-Følner set of trees $\mu$ with support contained in $\left\{R_{f}: f \in A\right\}$;

(2) if $\mu$ is a weighted $\varepsilon$-Følner set of trees and $K \varepsilon \leq 1$, then there is a weighted $K \varepsilon$-Følner set of trees $v$ which is supported on a subset of

$$
\{\partial T:(\mu(T)>0) \wedge(\partial T \text { is non trivial }) \wedge(\Gamma \text { acts properly on } T)\}
$$

Thus by (1), if $A \subseteq F$ is $K^{-(n+1)}$-Følner, then there is an $A^{\prime} \subseteq A$ such that $\left\{R_{f}\right.$ : $\left.f \in A^{\prime}\right\}$ is an $K^{-n}$-Følner set. By applying (2) $n$ times and observing that weighted Følner sets have non-empty supports, we have that there is an $f \in A$ such that $\partial^{n} R_{f}$ is non trivial. Let $k_{i}=\left|\partial^{n-i} R_{f}\right|$ and observe that by Lemma 5.4, $k_{0} \geq 4$ and $k_{i+1}>2^{k_{i}-2}$. It follows by induction that $\exp _{i}(0)+2<k_{i}$ and in particular that $R_{f}$ contains at least $\exp _{n}(0)$ elements.

By Theorem 1 and Proposition 2 of [1], if $f$ is in $F$, then the distance from $f$ to the identity is at least $(k-2) / 3$, where $k$ is the common cardinality of the trees in the reduced tree diagram for $f$. In particular, if $k$ is at least $3-$ the minimum cardinality of a tree in any diagram representing a non-identity element - then the distance is at least $k / 16$. It is easily verified that for all $n>0, \frac{1}{16} \exp _{4 n}(0) \geq \exp _{n}(0)$. If $K$ is a constant which satisfies the conclusion of Claim 5.14, then define $C=K^{4}$.

I now claim that if $A$ is $C^{-n}$ Følner, then $|A| \geq \exp _{n}(0)$. To see this, let $A \subseteq F$ be $C^{-n}$-Følner. By Lemma 3.15, there is a finite $A^{\prime} \subseteq F$ which is $\Gamma$-connected, $C^{-n}$-Følner, and satisfies $\left|A^{\prime}\right| \leq|A|$. Since $A^{\prime}$ is $K^{-4} n$-Følner, our choice of $K$ implies that $A^{\prime}$ has an element $a$ whose reduced tree diagram contains trees with at least $\exp _{4 n}(0)$ leaves. It follows that the distance from $a$ to the identity is at least $\frac{1}{16} \exp _{4 n}(0) \geq \exp _{n}(0)$. Since $A^{\prime}$ is $\Gamma$-connected, it must contain at least $\exp _{n}(0)$ elements and thus $|A| \geq\left|A^{\prime}\right| \geq \exp _{n}(0)$, establishing Theorem 1.1. 


\section{References}

[1] J. Burillo, S. Cleary, and M. I. Stein, Metrics and embeddings of generalizations of Thompson's group F. Trans. Amer. Math. Soc. 353 (2001), 1677-1689. Zbl 0989.20030 MR 1806724

[2] J. W. Cannon, W. J. Floyd, and W. R. Parry, Introductory notes on Richard Thompson's groups. Enseign. Math. (2) 42 (1996), 215-256. Zbl 0880.20027 MR 1426438

[3] A. Erschler, On isoperimetric profiles of finitely generated groups. Geom. Dedicata 100 (2003), 157-171. Zbl 1049.20024 MR 2011120

[4] A. Erschler, Piecewise automatic groups. Duke Math. J. 134 (2006), 591-613. Zbl 1159.20019 MR 2254627

[5] S. M. Gersten and John R. Stallings (eds.), Combinatorial group theory and topology. Ann. of Math. Stud. 111, Princeton University Press, Princeton, NJ, 1987. Zbl 0611.00010 MR 0895606

[6] R. I. Grigorchuk, Growth and amenability of a semigroup and its group of quotients. In Proceedings of the international symposium on the semigroup theory and its related fields (Kyoto, 1990), Shimane University, Matsue 1990, 103-108. Zbl 0726.43002 MR 1099852

[7] M. Gromov, Entropy and isoperimetry for linear and non-linear group actions. Groups Geom. Dyn. 2 (2008), 499-593. Zbl 05368764 MR 2442946

[8] A. L. T. Paterson, Amenability. Math. Surveys Monogr. 29, Amer. Math. Soc., Providence, RI, 1988. Zbl 0648.43001 MR 961261

[9] Letter from Richard Thompson to George Francis, dated September 26, 1973.

Received May 8, 2009; revised April 4, 2012

J. Tatch Moore, Department of Mathematics, Cornell University, Ithaca, NY 14853-4201, U.S.A.

E-mail: justin@math.cornell.edu 